\title{
Simulation of Concurrent Games in Distributed Systems
}

\author{
Alexey N. Ivutin ${ }^{1}$, Eugene V. Larkin², Yuriy I. Lutskov² \\ ${ }^{1}$ Department of Computer Technology, Tula State University, 92 Lenin Ave., 300012, Tula, Russia \\ ${ }^{2}$ Department of Robotics and Industry Mechanisation, Tula State University, 92 Lenin Ave., 300012 , \\ Tula, Russia
}

Keywords: competition, concurrent game, Petri-Markov net, winner, loser, distance, efficiency, penalty, participant, time, waiting time.

\begin{abstract}
This article looks into one of the methods of evaluation of competition results. It was assumed that participants run their distances within a probable time period with a known distribution. There were considered the Petri-Markov models of paired competition, plural and group competitions. The time densities and probabilities, with which the participants may win and/or lose the competition, are deduced. Also, the authors come out with the general expressions for the time densities and the probabilities of finishing the distance by an arbitrary number of participants and groups of participants.
\end{abstract}

\section{Introduction}

At present, games theory is widely used in industry, economy, military, computer science and other branches of knowledge as a powerful concept of system simulation. Originally, the game theory was developed based on static games with their payoff matrix and strategies of players, which result in winning or losing some resources. In computer science game theory is used for modelling the interaction inside networks, between a processor, computational modules, peripherals, and etc. [1]

Temporal aspects of game evolution, especially the evolution of a game in physical time, are considered insufficiently. At the same time, a concurrent game in temporal domain is the main process of every concurrent system. In this article we made the assumption that:

1.A certain quantity (number) of participants take part in a game;

2.A game develops in real physical time;

3.During a game, the participants "run" a certain "distance"; they start their distances at the same time, and winning or losing the game is understood as being the first or not the first to finish the distance;

4.Until the losers finish their distances, the winners receive penalties from all the losers, and a value of the penalty is distributed on time;

5.Time intervals, within which the participants run their distances, are random, and these intervals are calculated with accuracy to density of distribution.

The importance of developing an approach for modeling, specification, verification and synthesis of discrete event processes, with particular emphasis on computer operating systems, database management, concurrent programs, and distributed computing, has been recognized in the computer science community for well over a decade, and a diverse and extensive literature has been developed on this subject [2].

In this article the authors introduce the mathematical formalism that fully takes into account such features as a run-time accident and a possibility of simultaneous execution of groups of operators, as well as quasi-stochastic nature of the transitions in decision-making. This model is called PetriMarkov nets [3], and it assumes the composition of structures of parallel algorithms, stochastic-time parameters and logical conditions.

\section{Competition of J Participants}

Common Petri net model of $J$ participants competition is presented in fig 1 , where places $a_{1}, \ldots$, $a_{j}, \ldots, a_{J}$ simulate the process of distance running by the first, $\ldots, j$-th, ..., and $J$-th participant, 
respectively, transition $z_{1}$ simulates the start of the competition, and transition $z_{2}$ simulates the finishing of a distance.

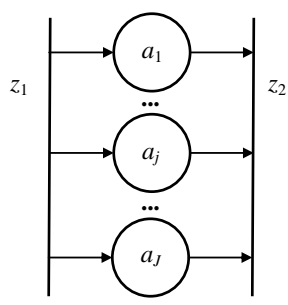

Fig. 1: Concurrency of $J$ participants

Let us take $J=2$ and split time intervals on periods $d t$. Then the result of the competition may be described with the semi-Markov process, which is shown in fig. 2.

In the semi-Markov process: the state $\alpha$ simulates the start of the competition; the state $\alpha_{w 1}$ simulates the winning of the competition by the first participant; the state $\alpha_{w 2}$ simulates the winning of the competition by the second participant; the state $\alpha_{w 1}$ simulates the draw in the competition; states $\beta_{-n},-\infty<-n<0$ simulate the situation, in which the first participant wins the competition with the result $t=n d t$; states $\beta_{n}, 0<n<\infty$, simulate the situation, in which the second participant wins the competition with the result $t=n d t$; and state $\beta_{0}$ simulates a draw result, probability of which is less, than probabilities of any other results.

Let us determine the time densities of achieving the absorbing states $\alpha_{w 1}, \alpha_{w 2}, \alpha_{d}$ from the initial state $\alpha$. The probability of the fact that within a period of time $t=n \Delta_{t}$ the state $\alpha_{w 1}$ will be achieved with a time lag defined by the state $\beta_{-n}$, is equal to $P_{-n}\left(t=n \Delta_{t}\right)=\left[1-F_{2}\left(n \Delta_{t}\right)\right] \cdot f_{1}\left(n \Delta_{t}\right) \Delta_{t}$, where $F_{2}\left(n \Delta_{t}\right)=\int_{0}^{n \Delta_{t}} f_{2}(\tau) d \tau$ - is the distribution function corresponding to density $f_{2}(t)$. The probability of the fact that within a period of time $t=n \Delta_{t}$ the state $\alpha_{w 2}$ will be achieved with a time lag defined by the state $\beta_{n}$, is equal to $P_{-n}\left(t=n \Delta_{t}\right)=\left[1-F_{1}\left(n \Delta_{t}\right)\right] \cdot f_{2}\left(n \Delta_{t}\right) \Delta_{t}$, where $F_{1}\left(n \Delta_{t}\right)=\int_{0}^{n \Delta_{t}} f_{1}(\tau) d \tau$. The probability of the fact that within a period of time $t=n \Delta_{t}$ the state $\alpha_{d}$ will be achieved with a time lag defined by the state $\beta_{0}$, is equal to $P_{0}\left(t=n \Delta_{t}\right)=f_{1}\left(n \Delta_{t}\right) \cdot f_{2}\left(n \Delta_{t}\right) \Delta_{t}^{2}$, and is much less probable than probabilities $P_{-n}\left(t=n \Delta_{t}\right)$ and $P_{n}\left(t=n \Delta_{t}\right)$. Weighted time densities of achievement of states $\alpha_{w 1}$ and $\alpha_{w 2}$ are defined as follows:

$$
\begin{aligned}
& h_{w 1}(t)=\lim _{\substack{n \rightarrow \infty \\
\Delta_{t} \rightarrow 0}} \frac{P_{-n}\left(t=n \Delta_{t}\right)}{\Delta_{t}}=\left[1-F_{2}(t)\right] \cdot f_{1}(t) ; \\
& h_{w 2}(t)=\lim _{\substack{n \rightarrow \infty \\
\Delta_{t} \rightarrow 0}} \frac{P_{n}\left(t=n \Delta_{t}\right)}{\Delta_{t}}=\left[1-F_{1}(t)\right] \cdot f_{2}(t) .
\end{aligned}
$$

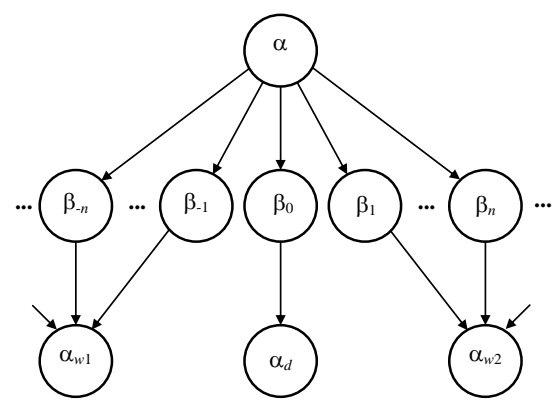

Fig. 2: Semi-Markov process for Petri net in fig. 1, when $\mathrm{J}=2$

So the sum of weighted densities (1) and (2) is equal to:

$$
h_{w 1}(t)+h_{w 2}(t)=\frac{d}{d t}\left\{1-\left[1-F_{1}(t)\right] \cdot\left[1-F_{2}(t)\right]\right\} .
$$


For a common case of concurrency of $J$ participants the weighted sum of densities may be obtained from (3) with the mathematical induction method:

$$
\sum_{j=1}^{J} h_{w j}(t)=\frac{d}{d t}\left\{1-\prod_{j=1}^{J}\left[1-F_{j}(t)\right]\right\},
$$

where $F_{j}(t)=\int_{0}^{t} f_{j}(\tau) d \tau$ - are the distribution functions corresponding to the densities $f_{j}(t), 1 \leq j \leq J$.

The probabilities of winning the competition by the $j$-th participant from (4) may be obtained as follows:

$$
p_{w j}=\int_{0}^{\infty} f_{j}(t) \cdot \prod_{\substack{k=1 \\ k \neq j}}^{J}\left[1-F_{k}(t)\right] d t, 1 \leq j \leq J .
$$

The time density of achievement of the transition $z_{2}$ by the participant-winner $j$ is defined as:

$$
f_{w j}(t)=\frac{\left(f_{j}(t) \cdot \prod_{\substack{k=1 \\ k \neq j}}^{J}\left[1-F_{k}(t)\right]\right)}{p_{w j}} .
$$

For example, let us look into the competition where $J$ participants are united into $K$ groups, with $J(k)$ participants in the $k$-th group, so that $\sum_{k=1}^{K} J(k)=J$.

In accordance with the Petri model, the time density of the distance completion by the whole $k$-th group is defined as:

$$
f_{k}(t)=\frac{d}{d t}\left[\prod_{\substack{k=1 \\ j=\sum_{n=1}^{k} J(n)+1}}^{\substack{k \\ n=1}} F_{j}(t)\right] .
$$

\section{Waiting Time}

Let us consider the situation, in which the first participant finishes the distance at the moment $\tau$ and is waiting until the second participant makes the finish. In this case we subtract the probable time of the first participant finishing the distance from the probable time of the second participant finishing the distance. The density of difference of the probable values is defined by the correlation integral, i.e. $\int_{0}^{\infty} f_{1}(\tau) f_{2}(t+\tau) d \tau$. The weighted time density of waiting by the first participant until the second participant finishes the distance is derived by means the truncation of a part with the negative argument from the correlation integral:

$$
h_{1 \rightarrow 2}(t)=\eta(t) \int_{0}^{\infty} f_{1}(\tau) f_{2}(t+\tau) d \tau .
$$

The probability of winning by the first participant is defined by the expression, derived from (3):

$$
p_{1 \rightarrow 2}=\int_{0}^{\infty} f_{1}(t) \cdot\left[1-F_{2}(t)\right] d t=1-\int_{0}^{\infty} f_{1}(t) \cdot F_{2}(t) d t=\int_{0}^{\infty} F_{1}(t) d F_{2}(t) .
$$

The density of waiting time for the case when the first participant finishes his distance the first is the following

$$
f_{1 \rightarrow 2}(t)=\frac{\eta(t) \int_{0}^{\infty} f_{1}(\tau) f_{2}(t+\tau) d \tau}{\int_{0}^{\infty} F_{1}(t) d F_{2}(t)},
$$


where $\eta(t)$ - is the Heaviside function.

It is necessary to say that expression (10) is not the commutative one, and in the general case:

$$
f_{2 \rightarrow 1}(t)=\frac{\eta(t) \int_{0}^{\infty} f_{2}(\tau) f_{1}(t+\tau) d \tau}{\int_{0}^{\infty} F_{2}(t) d F_{1}(t)} \neq f_{1 \rightarrow 2}(t) .
$$

\section{Evaluation of Competition Efficiency}

One of the most important factors of the competition simulation is the evaluation of its efficiency. The natural model of efficiency evaluation is a model, according to which the participant, who has finished the distance, receives the penalty from the losers until the losers finish the distance. In the proposed conception the competition proceeds within physical time, and there is an evaluation of the waiting time density, therefore it is natural to define the payoff matrix as follows:

$$
S(t)=\left\lfloor s_{j i}(t)\right] .
$$

where $s_{j i}(t)$ - is the "density of penalties", the value of which, at time $t$, is equal to a part of the payoff received by the $j$-th winner to the $i$-th loser.

In general, $s_{j i}(t) \neq s_{i j}(t)$, therefore matrix (12) is asymmetrical with respect to the main diagonal. As the participant, who finished the distance, cannot penalize himself, $s_{j j}(t)=0$.

The First and the simplest principle consists in the fact that the $l$-th group, which lost the competition, pays a penalty to the winning $k$-th group. In this case the "density of penalties" is evaluated as follows:

The group $k$ wins from the group $l$ a total penalty of:

$$
s_{k l}(t)=\sum_{\substack{k-1 \\ j=\sum_{n=1}^{k} J(n)+1}}^{k} \sum_{r=1}^{l-1} J(n)
$$

$$
s_{k l}^{+}=\int_{0}^{\infty} f_{k \rightarrow l}(t) \cdot s_{k l}(t) d t
$$

where $f_{k \rightarrow l}(t)$ is defined by expression (10); $f_{k}(t)$ and $f_{l}(t)$ in (10) are defined by expression (7).

The second and more complex principle consists in the fact that every participant in the $l$-th group, which lost the competition, pays a penalty to the participants of the winning $k$-th group individually. In this case, the time of waiting by the $k$-th group for the completion of the distance by the participants from the $l$-th group is determined with the expression, derivable from (10):

$$
f_{k \rightarrow j}(t)=\frac{\eta(t) \int_{0}^{\infty} f_{k}(\tau) f_{j}(t+\tau) d \tau}{\int_{0}^{\infty} F_{k}(t) d F_{j}(t)},
$$

where $\sum_{n=1}^{l-1} J(n)+1 \leq j \leq \sum_{n=1}^{l} J(n) ; f_{k}(\tau)$ is the time density, which is defined by expression (7).

The group $k$ wins from the group $l$ a total penalty of:

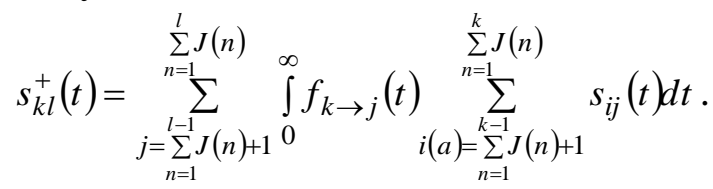

In the competition under examination there may be other principles of penalty payoff, for which other formulas of payoff evaluation are applied, but the general principles can be understood from the examples above. 


\section{Numerical Example}

As an example let us consider the case of paired competition, which is expressed by the PetriMarkov net, in which $\mathbf{J}=2$ and time densities $f_{1}(t)$, and $f_{2}(t)$ are equal to (fig. 3 a):

$$
f_{1}(t)=\left\{\begin{array}{l}
0, \text { when } 0 \leq t<0,5 ; \\
1_{1}, \text { when } 0,5 \leq t \leq 1,5 ; \quad f_{2}(t)=1_{1} \exp \left(-1_{2} \cdot t\right), \\
0, \text { when } t>1,5
\end{array}\right.
$$

where parameters $1_{1}$ and $1_{2}$ have the dimension of $1_{1}-\left[\frac{\text { prob. }}{\text { time }}\right] ; 1_{2}-\left[\frac{1}{\text { time }}\right]$.

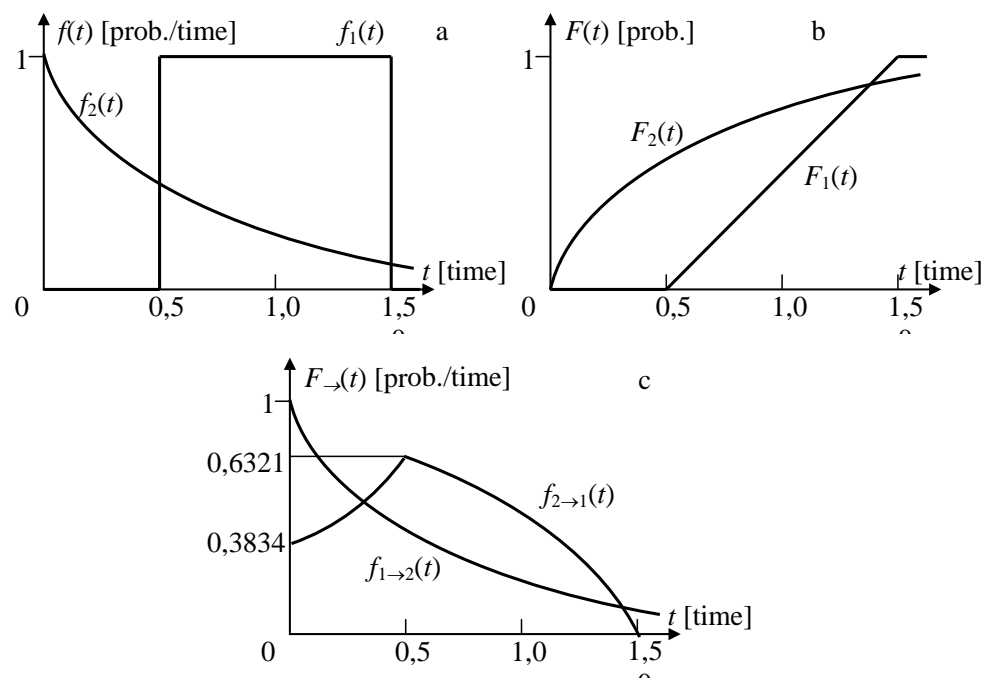

Fig. 3: Time densities and time distributions

It is obvious that $f_{1}(t)$, and $f_{2}(t)$ have the dimension of $\left[\frac{\text { prob. }}{\text { time }}\right]$. The expectations of time densities are quite equal, i.e. $T_{1}=T_{2}=1$ [time]. The distribution functions, corresponding to the time densities $f_{1}(t)$, and $f_{2}(t)$, and having the [prob.] dimension are as follows (fig. $3 \mathrm{~b}$ ):

$$
\begin{aligned}
& F_{1}(t)=\left\{\begin{array}{l}
0, \text { when } 0 \leq t<0,5 ; \\
t-0,6, \text { when } 0,5 \leq t \leq 1,5[\text { prob }] \\
1, \text { when } t>1,5 .
\end{array}\right. \\
& F_{2}(t)=1-\exp (-t)[\text { prob] } .
\end{aligned}
$$

In spite of equality of expectations for $f_{1}(t)$ and $f_{2}(t)$, the probabilities of winning by the participants 1 and 2, calculated based on expression (5), are quite different: $p_{w 1}=0.3834$ [prob]; $p_{w 2}=0.6166[$ prob $]$.

The waiting time densities, calculated based on the expression (10), are equal (fig. $3 \mathrm{c}$ ):

$$
\begin{gathered}
f_{1 \rightarrow 2}(t)=1_{1} \exp \left(-1_{2} \cdot t\right)\left[\frac{\text { prob. }}{\text { time }}\right] ; \\
f_{2 \rightarrow 1}(t)=\frac{1}{0,3834}\left\{\begin{array}{l}
0,3834 \cdot \exp \left(1_{2} \cdot t\right) \text { when } 0 \leq t \leq 0,5 ; \\
1-0,2231 \cdot \exp \left(1_{2} \cdot t\right) \text { when } 0,5<t \leq 1,5 .\left[\frac{\text { prob. }}{\text { time }}\right]
\end{array}\right] .
\end{gathered}
$$

If the density of penalties is equal to $s_{12}(t)=s_{21}(t)=\tilde{N} \cdot \exp (-c t)$, where $C\left[\frac{\text { doll. }}{\text { time. prob. }}\right]$ - is a coefficient; $c\left[\frac{1}{\text { time }}\right]$ - is a speed of decrease of demand on the results of winning, then the sum of penalties, which the first participant gets from the second one with the probability of 0,6166 , is equal to: 


$$
s_{1}^{+}=\tilde{N} \cdot 1_{1} \int_{0}^{\infty} \exp \left[-\left(1_{2}+c\right) \cdot t\right] d t=\frac{C \cdot 1_{1}}{1_{2}+c}[\text { doll. }] .
$$

The penalty, which the second participant gets from the first one with the probability of 0,3834 , is equal to:

$$
\begin{gathered}
s_{2}^{+}=\frac{C}{1_{2}+c}\left[1+1,5819 \cdot \exp 0,5\left(1_{2}-c\right)-0,5819 \cdot \exp 1,5\left(1_{2}-c\right)\right]+ \\
+\frac{2,6082 \cdot C}{c}(\exp 0,5 c-\exp 1,5 c) .
\end{gathered}
$$

In spite of equality of expectation of $f_{1}(t)$ and $f_{2}(t)$, the equality of penalty density sums, which a participant can potentially win from the partners, and the probabilities of winning are quite different, and these obstacles should be taken into account when planning the concurrent games.

\section{Conclusions}

We have presented a competition as a result of "running" by its participants of some distance during a probable time, which is defined with accuracy to distribution. It was shown that the mathematical apparatus of the Petri-Markov net is an efficient instrument for the simulation of such cases, which permits to describe all cases of a competition, from the paired to the group ones. Also, it permits to evaluate the efficiency of participation in a competition with this of that distribution of running the distance.

The time and stochastic characteristics were obtained in a general form. They are essential for planning the strategy and tactics of a competition, if a strategy/tactic may change time densities of running the distance by the participants. The following researches in this domain may be directed at working out the apparatus, which links the proposed method of simulation of a competition with the traditional game theory. Also, the method may be useful for solving the task of game optimization, which permits to generate the aim function or the restrictions for this task. The development of this method may be directed at working out a simple engineering method of efficiency calculation with the use of only numerical characteristics of time distributions.

\section{References}

[1] Chatterjee K., Jurdziński M., Henzinger T. Simple stochastic parity games // Lect. Notes Comput. Sci. 2003. Vol. 2803. P. 100-113.

[2] Heymann M. Concurrency and Discrete Event Control // IEEE Control Syst. Mag. 1990. Vol. 10. P. 103-112.

[3] Ivutin, A., Larkin, E. Estimation of Latency in Embedded Real-Time Systems // 3rd Mediterranean Conference on Embedded Computing (MECO) - 2014 June 15-19 2014 Budva, Montenegro, 2014 - P.236-239. 